\title{
Spin Hall and Spin Swapping Torques in Diffusive Ferromagnets
}

\author{
Christian Ortiz Pauyac, ${ }^{1,2}$ Mairbek Chshiev, ${ }^{2}$ Aurelien Manchon, ${ }^{1,3}$ and Sergey A. Nikolaev ${ }^{4,2, *}$ \\ ${ }^{1}$ Physical Science and Engineering Division (PSE), King Abdullah University of Science and Technology (KAUST), \\ Thuwal 23955-6900, Saudi Arabia \\ ${ }^{2}$ Univ. Grenoble Alpes, CNRS, CEA, Grenoble INP, INAC-SPINTEC, 38000 Grenoble, France \\ ${ }^{3}$ Computer, Electrical and Mathematical Science and Engineering (CEMSE), \\ King Abdullah University of Science and Technology (KAUST), Thuwal 23955-6900, Saudi Arabia \\ ${ }^{4}$ Ural Federal University, 10 Mira Street, 620002 Ekaterinburg, Russia
}

(Received 8 December 2017; published 27 April 2018)

\begin{abstract}
A complete set of the generalized drift-diffusion equations for a coupled charge and spin dynamics in ferromagnets in the presence of extrinsic spin-orbit coupling is derived from the quantum kinetic approach, covering major transport phenomena, such as the spin and anomalous Hall effects, spin swapping, spin precession, and relaxation processes. We argue that the spin swapping effect in ferromagnets is enhanced due to spin polarization, while the overall spin texture induced by the interplay of spin-orbital and spin precession effects displays a complex spatial dependence that can be exploited to generate torques and nucleate or propagate domain walls in centrosymmetric geometries without the use of external polarizers, as opposed to the conventional understanding of spin-orbit mediated torques.
\end{abstract}

DOI: 10.1103/PhysRevLett.120.176802

Introduction.-The exploitation of spin-orbit coupling (SOC) effects to probe and control the magnetization in nanodevices has been extensively studied, uncovering many physical phenomena, such as the anomalous Hall effect [1], the spin Hall effect [2], tunneling anisotropic magnetoresistance [3], electrically controlled perpendicular magnetic anisotropy [4], and relativistic spin torques [5-7]. Relativistic spin torques observed in multilayers comprising ferromagnets and normal metals display both spin-orbit torques induced by the interfacial inverse spin-galvanic effect [6] and spin-transfer torques associated with the spin Hall effect in an adjacent nonmagnetic layer [7]. Spin-orbit torques generated in a single ferromagnetic layer are of great importance in enabling electrical control of the magnetization without the use of external polarizers, and they offer many promising advantages compared to spintransfer torques, such as high scalability and stability. Thus, finding novel routes to excite magnetization dynamics by means of spin-orbit torques is essential for realizing highperformance spintronic devices.

Meanwhile, new ways to generate spin accumulation are also of great interest. Recently, a new mechanism referred to as spin swapping, which converts a primary spin current into a secondary spin current with interchanged spin and flow directions, was proposed to exist in normal metals and semiconductors in the presence of spin-orbit coupled impurities [8]. However, whether spin swapping in ferromagnets can produce a measurable effect remains an open question that has not yet been addressed. On the one hand, the exchange magnetic field present in ferromagnets tends to destroy the induced spin accumulation. On the other hand, SOC not only acts constructively in generating spin accumulation, it also leads to spin-memory loss [9]. Overall, the possibility to employ these effects in ferromagnets strongly depends on the transport regime as a function of many parameters describing a given system.

In this Letter, we explore the nature of the extrinsic spin Hall and spin swapping effects in diffusive ferromagnets and demonstrate that these effects can offer potential advantages, in contrast to noncentrosymmetric magnetic multilayers involving heavy metals. To this end, we develop a set of coupled spin-charge diffusive equations by using the nonequilibrium Green's function formalism and taking into account scattering off the impurity induced SOC potential. Based on these equations, we proceed to study the interplay between spin-orbital and spin precession effects that can be used to demonstrate current-driven manipulation of the magnetization in centrosymmetric magnets.

Derivation of the coupled spin-charge drift-diffusion equation.-We consider a single ferromagnetic layer in the standard $s$ - $d$ model [10] defined as $\hat{\mathcal{H}}=\left(\hat{\boldsymbol{p}}^{2} / 2 m\right) \hat{\sigma}_{0}+$ $J \hat{\boldsymbol{\sigma}} \cdot \boldsymbol{m}+\hat{\mathcal{H}}_{\text {imp }}$, where $m$ is the effective electron's mass, $\hat{\boldsymbol{p}}$ is the momentum operator, $J$ is the exchange coupling, $\hat{\sigma}_{0}$ is the identity matrix, $\hat{\boldsymbol{\sigma}}$ is the Pauli matrix vector, and $\boldsymbol{m}$ is the spatial magnetization profile. Here, the third term stands for the impurity potential given by randomly distributed $N$ impurities $\boldsymbol{R}_{j}, \hat{\mathcal{H}}_{\text {imp }}=\sum_{j}^{N}\left\{V\left(\boldsymbol{r}-\boldsymbol{R}_{j}\right) \hat{\sigma}_{0}+\left[\xi_{\mathrm{So}} /\left(\hbar k_{F}^{2}\right)\right] \hat{\boldsymbol{\sigma}}\right.$. $\left.\left[\boldsymbol{\nabla} V\left(\boldsymbol{r}-\boldsymbol{R}_{j}\right) \times \hat{\boldsymbol{p}}\right]\right\}$, where $V\left(\boldsymbol{r}-\boldsymbol{R}_{j}\right)=v_{i} \delta\left(\boldsymbol{r}-\boldsymbol{R}_{j}\right)$ is the on-site impurity potential, $\xi_{\mathrm{SO}}$ is the SOC parameter (defined as a dimensionless quantity), and $k_{F}$ is the Fermi wave vector. In the Keldysh formalism for an 
interacting system driven out of equilibrium, the Dyson equation for the nonequilibrium Green's function $\hat{G}^{K}$ is written as

$$
\left[\hat{G}^{R}\right]^{-1} \circ \hat{G}^{K}-\hat{G}^{K} \circ\left[\hat{G}^{A}\right]^{-1}=\hat{\Sigma}^{K} \circ \hat{G}^{A}-\hat{G}^{R} \circ \hat{\Sigma}^{K},
$$

where $\hat{G}^{i} \equiv \hat{G}^{i}\left(\boldsymbol{r}, t ; \boldsymbol{r}^{\prime}, t^{\prime}\right) \quad$ and $\quad \hat{\Sigma}^{i} \equiv \hat{\Sigma}^{i}\left(\boldsymbol{r}, t ; \boldsymbol{r}^{\prime}, t^{\prime}\right) \quad$ with $i=K, R, A$ are the real space, real time Keldysh, retarded, and advanced Green's functions and self-energies, respectively, and $\left[\hat{G}^{R(A)}\right]^{-1}=\left[\hat{G}_{0}\right]^{-1}-\hat{\Sigma}^{R(A)}$, where $\hat{G}_{0}^{-1}=i \hbar \partial_{t}-\hat{\mathcal{H}}$ is a noninteracting Green's function for the system without impurities [11,12]. Having applied the Wigner transformation $\quad \hat{G}^{K}\left(\boldsymbol{r}, t ; \boldsymbol{r}^{\prime}, t^{\prime}\right)=\int(d E / 2 \pi)\left[d \boldsymbol{k} /(2 \pi)^{3}\right]$ $e^{i \mathbf{k} \cdot\left(\mathbf{r}-\mathbf{r}^{\prime}\right)-i(E / \hbar)\left(t-t^{\prime}\right)} \hat{g}_{\mathbf{k}, E}^{K}(\mathbf{R}, T)$, with $\boldsymbol{R}=\left(\boldsymbol{r}+\boldsymbol{r}^{\prime}\right) / 2$ and $T=\left(t+t^{\prime}\right) / 2$, we employ the so-called gradient approximation to linearize convolutions (the open circles) in the Dyson equation (1) and obtain the following quantum kinetic equation for the distribution function $\hat{g}_{k}=$ $i \int(d E / 2 \pi) \hat{g}_{k, E}^{K}(\boldsymbol{R}, T)$ :

$$
\hbar \partial_{T} \hat{g}_{\boldsymbol{k}}-i\left[\hat{g}_{\boldsymbol{k}}, J \hat{\boldsymbol{\sigma}} \cdot \boldsymbol{m}\right]+\frac{\hbar^{2}}{m}\left(\boldsymbol{k} \cdot \boldsymbol{\nabla}_{\boldsymbol{R}}\right) \hat{g}_{\boldsymbol{k}}=\int \frac{d E}{2 \pi} \mathcal{C},
$$

where $\mathcal{C}=\left(\hat{\Sigma}^{K} \hat{G}^{A}-\hat{G}^{R} \hat{\Sigma}^{K}\right)+\left(\hat{\Sigma}^{R} \hat{g}^{K}-\hat{g}^{K} \hat{\Sigma}^{A}\right)$ is the collision integral that accounts for the scattering and relaxation events, respectively. Scattering off the impurity potential in the right-hand side of Eq. (2) is considered up to third order by impurity averaging over disorder with concentration $n_{i}$ so that the spin-dependent momentum and spin-flip relaxations and the side-jump [13], spin swapping, and skewscattering [14] processes are properly taken into account. In the diffusive limit, where the mean free path is much smaller than the size of the system, one can partition the distribution function $\hat{g}_{k}=\hat{\sigma}_{0}-2 \hat{h}_{k}$ into the isotropic charge $\mu_{c}$, spin $\boldsymbol{\mu}$, and anisotropic $\hat{j}$ components, $\hat{h}_{k}=$ $\mu_{c} \hat{\sigma}_{0}+\boldsymbol{\mu} \cdot \hat{\boldsymbol{\sigma}}+\hat{\boldsymbol{j}} \cdot \check{\boldsymbol{k}}$, where $\check{\boldsymbol{k}}=\boldsymbol{k} /|\boldsymbol{k}|$ [15]. First, integrating Eq. (2) multiplied by $\breve{k}$ over the Brillouin zone gives us the corresponding expression for $\hat{\boldsymbol{j}} \equiv \hat{\boldsymbol{j}}\left(\mu_{c}, \boldsymbol{\mu}\right)$. Second, integrating Eq. (2) itself leads to the generalized continuity equation for the charge $\mu_{c}$ and spin $\boldsymbol{\mu}$ densities, so their time dependence is given as a divergence of the charge $j^{C}$ and spin $\boldsymbol{J}_{j}^{S}$ (its $j$ th spin component) currents, respectively. We refer the reader to Ref. [16] for more details concerning the derivation. Finally, in the weak exchange coupling limit ( $J \ll \varepsilon_{F}$, where $\varepsilon_{F}$ is the Fermi energy), the resulting driftdiffusion equations up to leading order in the exchange interaction and SOC have the following form:

$$
\partial_{T} \mu_{c}=D \nabla^{2}\left[\mu_{c}+\beta \boldsymbol{\mu} \cdot \boldsymbol{m}\right]=-\boldsymbol{\nabla} \cdot \boldsymbol{j}^{C},
$$

$$
\partial_{T} \boldsymbol{\mu}=-\boldsymbol{\nabla} \cdot \boldsymbol{J}^{S}+\frac{1}{\tau_{L}} \boldsymbol{m} \times \boldsymbol{\mu}+\frac{1}{\tau_{\phi}} \boldsymbol{m} \times(\boldsymbol{m} \times \boldsymbol{\mu})-\frac{1}{\tau_{\mathrm{sf}}} \boldsymbol{\mu},
$$

$$
\begin{aligned}
\boldsymbol{J}_{j}^{S} / D= & \tilde{\boldsymbol{J}}_{j}^{S} / D+\nabla \times\left[\alpha_{\mathrm{sj}} \boldsymbol{e}_{j}\left(2 \mu_{c}-\beta \boldsymbol{\mu} \cdot \boldsymbol{m}\right)+\alpha_{\mathrm{sk}} \boldsymbol{e}_{j}\left(\mu_{c}-\beta \boldsymbol{\mu} \cdot \boldsymbol{m}\right)-\alpha_{\mathrm{sw}} \boldsymbol{e}_{j} \times\left(\boldsymbol{\mu}-\beta \mu_{c} \boldsymbol{m}\right)+\frac{\tau_{0}}{\tau_{L}}\left(\alpha_{\mathrm{sj}}+\alpha_{\mathrm{sk}}\right)\left(\boldsymbol{e}_{j} \times \boldsymbol{m}\right) \mu_{c}\right. \\
& \left.+\left(\alpha_{\mathrm{sj}} \beta+\alpha_{\mathrm{sk}} \beta-\alpha_{\mathrm{sw}} \frac{\tau_{0}}{\tau_{L}}\right)\left(\boldsymbol{e}_{j} \times \boldsymbol{m}\right) \times \boldsymbol{\mu}-\left(\alpha_{\mathrm{sw}} \frac{\tau_{0}}{\tau_{L}}+\alpha_{\mathrm{sj}} \beta+\alpha_{\mathrm{sk}} \beta\right) \boldsymbol{e}_{j} \times(\boldsymbol{m} \times \boldsymbol{\mu})\right]
\end{aligned}
$$

where $\quad \tilde{\boldsymbol{j}}^{C}=-D \boldsymbol{\nabla}\left(\mu_{c}+\beta \boldsymbol{m} \cdot \boldsymbol{\mu}\right) \quad$ and $\quad \tilde{\boldsymbol{J}}_{j}^{S} / D=-\boldsymbol{\nabla}\left(\mu_{j}+\right.$ $\left.\beta \mu_{c} m_{j}\right)+\left(\tau_{0} / \tau_{L}\right) \boldsymbol{\nabla}(\boldsymbol{m} \times \boldsymbol{\mu})_{j}+\left(\tau_{0} / \tau_{\phi}\right) \boldsymbol{\nabla}[\boldsymbol{m} \times(\boldsymbol{m} \times \boldsymbol{\mu})]_{j}$ are the charge and spin currents in the absence of SOC, respectively; $\beta=\left(J / 2 \varepsilon_{F}\right)$ is the polarization factor; $\alpha_{\mathrm{sw}}=$ $\left[\left(2 \xi_{\mathrm{SO}}\right) / 3\right], \alpha_{\mathrm{sj}}=\left[\xi_{\mathrm{SO}} /\left(l_{F} k_{F}\right)\right]$, and $\alpha_{\mathrm{sk}}=\left[\left(v_{i} m k_{F} \xi_{\mathrm{SO}}\right) /\right.$ $\left.\left(3 \pi \hbar^{2}\right)\right]$ are the dimensionless spin swapping, side-jump, and skew-scattering coefficients, respectively; $D=$ $\left[\left(\tau_{0} v_{F}^{2}\right) / 3\right]$ is the diffusion coefficient, $v_{F}$ is the Fermi velocity, and $l_{F}=\tau_{0} v_{F}$ is the mean free path; $\boldsymbol{e}_{j}$ is the unit vector along the $j$ axis. Here, $\left(1 / \tau_{0}\right)=\left[\left(2 \pi v_{i}^{2} n_{i} D_{0}\right) / \hbar\right]$ is the spin-independent relaxation time, where $D_{0}=$ $\left[\left(m k_{F}\right) /\left(2 \pi^{2} \hbar^{2}\right)\right]$ is the spin-independent density of states, $\left(1 / \tau_{\mathrm{sf}}\right)=\frac{8}{9}\left(\xi_{\mathrm{SO}}^{2} / \tau_{0}\right)$ is the spin-flip relaxation time, $\left(1 / \tau_{L}\right)=(2 J / \hbar)$ is the spin precession time around the magnetization, known as the Larmor precession time, and $\left(1 / \tau_{\phi}\right)=\left[\left(4 J^{2} \tau_{0}\right) / \hbar^{2}\right]$ refers to the spin dephasing term. The set of drift-diffusion equations (3)-(6) is the central result of this Letter. On the one hand, in the absence of SOC, our approach is in line with the generalized driftdiffusion theory [17], which captures the main features of the transverse spin transport in ferromagnets, such as the Larmor precession and spin dephasing terms. On the other hand, in the case of normal metals $\left(\beta \rightarrow 0, \tau_{L} \rightarrow \infty\right.$, $\tau_{\phi} \rightarrow \infty$ ), our equations are in agreement with Shen et al. [18], while some other works fail to include the correct symmetry of the spin swapping term [16,19-21]. In the presence of both the exchange interaction and the extrinsic 
SOC, the anomalous Hall effect is present in Eq. (5) (the second and third terms) from both the side-jump and skewscattering processes [22], while spin polarization and spin precession give rise to additional terms to the anomalous charge and spin currents in Eqs. (5) and (6). Overall, the resulting charge and spin accumulation profiles appear to be much more complex than for normal metals. In magnetic systems with SOC, the competition between these effects is governed by the ratio of the corresponding characteristic lengths: spin precession, spin dephasing, and spin diffusion lengths. In ferromagnets with a strong exchange coupling, where the spin dephasing length is shorter than the spin-flip relaxation, the spin Hall and spin swapping effects are expected to vanish far from the interface, as the strong exchange field tends to destroy induced spin currents. Consequently, any transverse spin component will eventually align or antialign with the magnetization [23]. By contrast, in the weak exchange coupling limit $\left(J \ll \varepsilon_{F}\right)$, the spin dephasing length is larger or comparable to the spinflip relaxation, and these coupled effects can become prominent. Finally, it is worth pointing out that the resulting equations can be extended to include electromagnetic fields and gradient corrections in the case of a nonuniform magnetization profile [24]. While we believe that the latter are of less importance in smoothly varying magnetic textures, electric fields must be treated more cautiously. For example, it was argued that in normal metals the spin Hall effect is enhanced and spin swapping is almost compensated for when taking into account an electric-field induced spin-orbit coupling [18]. Even though in diffusive ferromagnets these effects may be more involved, we expect our results to hold qualitatively in the presence of electric fields.
Spin accumulation profile.-To study the interplay of the effects in question, the drift-diffusion equations (3)-(6) can be solved numerically by putting $\boldsymbol{m}$ along the $y$ axis and imposing adequate boundary conditions. Namely, we put $\boldsymbol{J}_{j}^{S}=0$ at the boundary to suppress interfacial spin-flip processes and let the spin accumulation build up at the edges, while the charge densities at the edges in the propagation direction are $\mu_{c L}=k_{F}^{2} / 2 \pi$ and $\mu_{c R}=0$ to enable current flow though the device. The results calculated for the rectangular geometry are shown in Fig. 1. In the absence of SOC, the spin current density $J_{x y}^{S} \sim \nabla_{x} \mu_{y}$ is induced in the magnetic layer and, due to Larmor precession, the spin accumulation $\mu_{y}$ is localized at the normal metal-ferromagnet interfaces along the transport direction [Fig. 1(a)], in agreement with the Valet-Fert theory [25]. When the impurity induced SOC is present, the transverse spin accumulation is expected to build up at the lateral edges. First off, let us analyze additional contributions to the spin swapping term. From Eq. (6), the "spin swapping" spin current has the following form:

$$
\begin{aligned}
J_{i j}^{\mathrm{sw}} / D= & \alpha_{\mathrm{sw}}\left(\nabla_{j} \mu_{i}-\delta_{i j} \nabla_{k} \mu_{k}\right)+\alpha_{\mathrm{sw}} \beta\left(\delta_{i j} m_{k} \nabla_{k}-m_{i} \nabla_{j}\right) \mu_{c} \\
& +\left(\alpha_{\mathrm{sj}} \frac{\tau_{0}}{\tau_{L}}+\alpha_{\mathrm{sk}} \frac{\tau_{0}}{\tau_{L}}\right)\left(\delta_{i j} m_{k} \nabla_{k}-m_{i} \nabla_{j}\right) \mu_{c}
\end{aligned}
$$

where summation over repeated indexes is implied. As seen, the resulting current meets symmetry requirements, $J_{i j}^{\mathrm{sW}} \sim \tilde{J}_{j i}^{S}-\delta_{i j} \tilde{J}_{k k}^{S}$, where $\tilde{J}_{j i}^{S}$ is the primary spin current $[8,16]$. In normal metals, owing to the spin swapping mechanism, $\tilde{J}_{j i}^{S} \sim \nabla_{j} \mu_{i}$ gives rise to the secondary spin

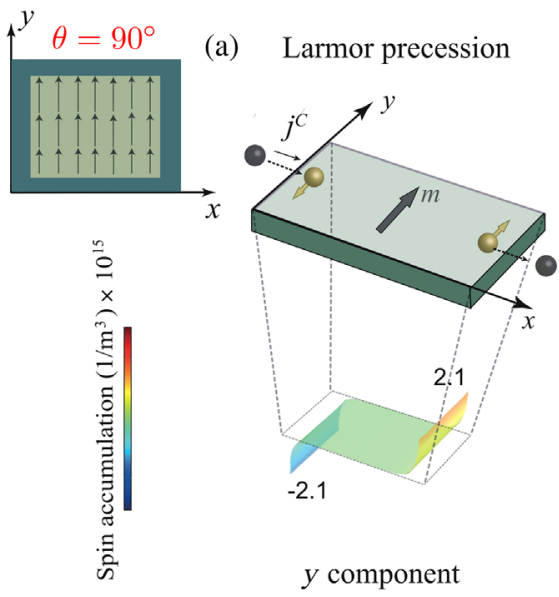

(b)

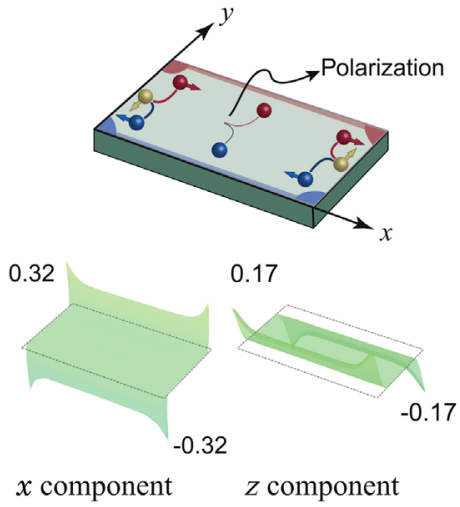

(c)

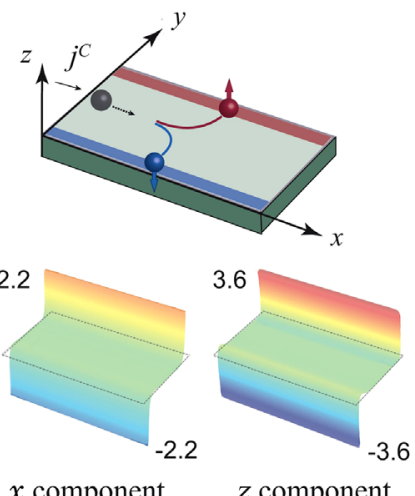

(d)

Total

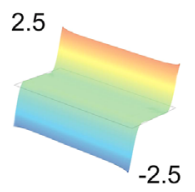

$x$ component

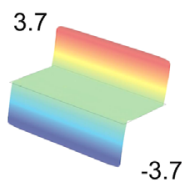

$z$ component

FIG. 1. Spin accumulation profiles $\mu_{x}, \mu_{y}$, and $\mu_{z}$ as calculated for the rectangular ferromagnetic geometry of $100 \times 50 \mathrm{~nm}^{2}$ (the light area) with a normal metal at the boundaries (the dark area). (a) $\mu_{y}$ in the case of Larmor precession only. (b) $\mu_{x}$ and $\mu_{z}$ when only the spin swapping and Larmor precession terms are considered. (c) $\mu_{x}$ and $\mu_{z}$ when only the spin Hall effect and Larmor precession are considered. (d) The full drift-diffusion equations. Here, the spin current polarized along the $y$ axis is flowing along the $x$ axis, $\varepsilon_{F}=0.7 \mathrm{eV}, J=0.02 \mathrm{eV}$, and $\xi_{\mathrm{SO}}=0.3$. The spin diffusion length is $l_{\mathrm{sf}}=\sqrt{D \tau_{\mathrm{sf}}}=5 \mathrm{~nm}$, the Larmor precession length is $l_{L}=\sqrt{D \tau_{L}}=2.6 \mathrm{~nm}$, the spin dephasing length is $l_{\phi}=\sqrt{D \tau_{\phi}}=4.8 \mathrm{~nm}$, the mean free path is $l_{F}=\tau_{0} v_{F}=2.5 \mathrm{~nm}$, the Fermi velocity is $v_{F}=5 \times 10^{5} \mathrm{~m} / \mathrm{s}$, and the Fermi wave vector is $k_{F}=4.3 \mathrm{~nm}^{-1}$. Grey and yellow spheres denote nonpolarized and spinpolarized charge currents, while red and blue spheres denote the spin-up and spin-down components, respectively. 
current $J_{i j}^{\mathrm{sw}} \sim \alpha_{\mathrm{sw}}\left(\nabla_{j} \mu_{i}-\delta_{i j} \nabla_{k} \mu_{k}\right)$, and the generated spin accumulation $\mu_{i}$ decays over a length scale given by the spin-flip relaxation length and survives only close to the interface [20]. However, in ferromagnets an additional nonvanishing spin accumulation $\sim \alpha_{\mathrm{sw}} \beta$ builds up due to spin polarization and develops smoothly at the lateral edges [Fig. 1(b), the $x$ component]. Interestingly, there is an extra term coming from the spin Hall effect and the precessional motion [the third term in Eq. (7)], which also exhibits the symmetry of the spin swapping current, even though it is not actually attributed to the spin swapping effect itself. The rest of the effects are related to Larmor precession. For example, let us consider a toy model where $\boldsymbol{m}$ points along the $y$ axis and the spin Hall effect, spin polarization, and higher-order terms to the precessional motion are discarded, so the spin current density in Eq. (6) is reduced to

$$
\begin{gathered}
\frac{J_{y x}^{S}}{D} \sim-\nabla_{y} \mu_{x}-\frac{\tau_{0}}{\tau_{L}} \nabla_{y} \mu_{z}+\alpha_{\mathrm{sw}} \nabla_{x} \mu_{y}, \\
\frac{J_{y z}^{S}}{D} \sim-\nabla_{y} \mu_{z}+\frac{\tau_{0}}{\tau_{L}} \nabla_{y} \mu_{x} .
\end{gathered}
$$

As one can see, Eq. (8) features the spin swapping effect. However, owing to Larmor precession, the second term in Eq. (9) couples $J_{y x}^{S}$ to $J_{y z}^{S}$ and builds up $\mu_{z}$, which is eventually related to the third term in Eq. (8) [Fig. 1(b), the $z$ component]. Similar results are obtained if we neglect spin swapping and focus on the spin Hall effect instead, where the spin accumulations do not vanish far from the interfaces [Fig. 1(c)]. If we solve our toy models separately for the spin swapping and side-jump terms, the following relation holds for the maxima of the corresponding spin accumulations:

$$
\frac{\mu_{x}}{\mu_{z}} \propto \frac{\alpha_{\mathrm{sw}} \beta}{\alpha_{\mathrm{sj}}}=\frac{1}{3} l_{F} k_{F} \beta .
$$

It follows that spin swapping is about 1 order of magnitude smaller than the spin Hall effect (as also seen in Fig. 1). While spin swapping scales with the momentum memory loss and is more pronounced when the mean free path is long enough [21], in diffusive ferromagnets it never becomes dominant over the spin Hall effect.

Current-driven magnetization switching.-We propose a possible way to exploit the spin Hall and spin swapping effects to reversibly control the magnetization in centrosymmetric ferromagnets that can be realized even in the absence of adjacent nonmagnetic layers. Normally, spinorbit torques are observed in ferromagnetic films lacking inversion symmetry through the Rashba effect, which is essentially inherent to noncentrosymmetric structures [6]. However, geometry itself can play an important role building up distorted spin accumulation profiles and giving rise to nonzero local spin-orbit torques. For example, let us

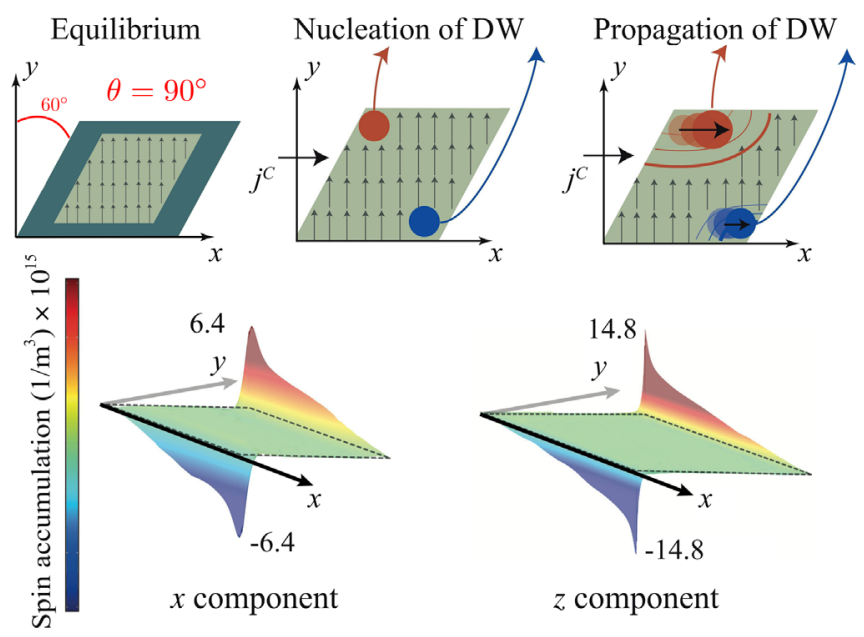

FIG. 2. Spin accumulation profiles $\mu_{x}$ (bottom-left panel) and $\mu_{z}$ (bottom-right panel), as calculated for the diamond-shaped geometry of $100 \times 50 \mathrm{~nm}^{2}$. The top panels describe the idea of nucleation and propagation of reversed magnetic domains. Red and blue circles correspond to the spin-up and spin-down components of the spin accumulation, respectively; black arrows show the magnetization pointed along the $y$ axis. The rest of the calculation parameters are given in Fig. 1.

consider a centrosymmetric diamond-shaped geometry. The resulting spin accumulation presented in Fig. 2 turns out to be highly asymmetric (while the net spin accumulation is zero) and peaks at the opposite edges that can be used to nucleate reversed magnetic domains. Once nucleated at the corners, the flowing current can either expand or shrink the reversed magnetic domain by currentdriven domain wall motion, as shown in the top panels of Fig. 2. A similar scenario in controlling the magnetization (albeit without considering the spin Hall or spin swapping mechanisms) was studied in Ref. [26].

Conclusion.-We derived a complete set of drift-diffusion equations for the coupled charge and spin transport in diffusive ferromagnets in the presence of extrinsic SOC. While combining major effects, such as the spin and inverse spin Hall effects, anomalous Hall effect and spin swapping, these equations reveal some intriguing new features. In particular, we showed that in ferromagnets the resulting spin accumulation exhibits a complex spatial profile, where the spin swapping effect is enhanced due to spin polarization and spin precession gives rise to additional contributions to the anomalous charge and spin currents. These effects can be employed to generate spin-orbit mediated torques and reversibly control the magnetization in centrosymmetric structures. Our results call for experimental approbation in current-driven magnetization dynamics, where suitable materials may include magnetic alloys with heavy impurities, such as $\mathrm{Co} / \mathrm{Pt}, \mathrm{Fe} / \mathrm{Au}$ [27], and $\mathrm{CuMn} / \mathrm{Pt}$ [28].

A. $\mathrm{M}$ and C. O.P were supported by King Abdullah University and Technology (KAUST). M. C. and S. A. N. were supported by the program "Emergence et partenariat 
stratégique" at the Univ. Grenoble Alpes. S. A. N. acknowledges support from PRESTIGE Postdoctoral Fellowship Programme, cofunded by the European Union. S. A. N. acknowledges support from the Act 211 Government of the Russian Federation, Contract No. 02.A03.21.0006.

C. O.P. and S. A. N. contributed equally to this work. *saishi@inbox.ru

[1] N. Nagaosa, J. Sinova, S. Onoda, A. H. McDonald, and N. P. Ong, Rev. Mod. Phys. 82, 1539 (2010).

[2] M. I. Dyakonov and V. I. Perel, Sov. Phys. JETP Lett. 13, 467 (1971); Phys. Lett. 35A, 459 (1971); J. E. Hirsch, Phys. Rev. Lett. 83, 1834 (1999).

[3] C. Gould, C. Ruster, T. Jungwirth, E. Girgis, G. M. Schott, R. Giraud, K. Brunner, G. Schmidt, and L. W. Molenkamp, Phys. Rev. Lett. 93, 117203 (2004); H. Saito, S. Yuasa, and K. Ando, Phys. Rev. Lett. 95, 086604 (2005); L. Gao, X. Jiang, S. H. Yang, J. D. Burton, E. Y. Tsymbal, and S. S. P. Parkin, Phys. Rev. Lett. 99, 226602 (2007).

[4] C.-G. Duan, J. P. Velev, R. F. Sabirianov, Z. Zhu, J. Chu, S. S. Jaswal, and E. Y. Tsymbal, Phys. Rev. Lett. 101, 137201 (2008); M. K. Niranjan, C. G. Duan, S. S. Jaswal, and E. Y. Tsymbal, Appl. Phys. Lett. 96, 222504 (2010); B. Dieny and M. Chshiev, Rev. Mod. Phys. 89, 025008 (2017).

[5] I. M. Miron, K. Garello, G. Gaudin, P.-J. Zermatten, M. V. Costache, S. Auffret, S. Bandiera, B. Rodmacq, A. Schuhl, and P.Gambardella, Nature (London) 476, 189-193 (2011).

[6] I. M. Miron, G. Gaudin, S. Auffret, B. Rodmacq, A. Schuhl, S. Pizzini, J. Vogel, and P. Gambardella, Nat. Mater. 9, 230 (2010); A. Manchon and S. Zhang, Phys. Rev. B 78, 212405 (2008).

[7] L. Liu, C.-F. Pai, Y. Li, H. W. Tseng, D. C. Ralph, and R. A. Buhrman, Science 336, 555 (2012).

[8] M. B. Lifshits and M. I. Dyakonov, Phys. Rev. Lett. 103, 186601 (2009).

[9] J. Fabian, A. Matos-Abiague, C. Ertler, P. Stano, and I. Žutić, Acta Phys. Slovaca 57, 565 (2007); J.-C. Rojas-Sánchez, N. Reyren, P. Laczkowski, W. Savero,
J.-P. Attané, C. Deranlot, M. Jamet, J.-M. George, L. Vila, and H. Jaffrès, Phys. Rev. Lett. 112, 106602 (2014).

[10] S. P. Shubin and S. V. Vonsovski, Proc. R. Soc. A 145, 159 (1934).

[11] J. Rammer and H. Smith, Rev. Mod. Phys. 58, 323 (1986).

[12] J. Rammer, Quantum Transport Theory (Westview Press, Boulder, 2004).

[13] L. Berger, Phys. Rev. B 2, 4559 (1970); S. K. Lyo and T. Holstein, Phys. Rev. Lett. 29, 423 (1972).

[14] S. Takahashi and S. Maekawa, J. Phys. Soc. Jpn. 77, 031009 (2008).

[15] R. V. Shchelushkin and Arne Brataas, Phys. Rev. B 71, 045123 (2005); 73, 169907 (2006).

[16] See Supplemental Material at http://link.aps.org/ supplemental/10.1103/PhysRevLett.120.176802 for a detailed derivation of drift-diffusion equations.

[17] C. Petitjean, D. Luc, and X. Waintal, Phys. Rev. Lett. 109, 117204 (2012).

[18] K. Shen, R. Raimondi, and G. Vignale, Phys. Rev. B 90, 245302 (2014); 92, 035301 (2015).

[19] R. V. Shchelushkin and A. Brataas, Phys. Rev. B 72, 073110 (2005).

[20] H. B. M. Saidaoui, Y. Otani, and A. Manchon, Phys. Rev. B 92, 024417 (2015).

[21] H. B. M. Saidaoui and A. Manchon, Phys. Rev. Lett. 117, 036601 (2016).

[22] W.-K. Tse and S. D. Sarma, Phys. Rev. Lett. 96, 056601 (2006); A. Crepieux and P. Bruno, Phys. Rev. B 64, 014416 (2001); V. K. Dugaev, A. Crepieux, and P. Bruno, Phys. Rev. B 64, 104411 (2001).

[23] T. Taniguchi, J. Grollier, and M. D. Stiles, Phys. Rev. Applied 3, 044001 (2015).

[24] S. A. Nikolaev (unpublished).

[25] T. Valet and A. Fert, Phys. Rev. B 48, 7099 (1993).

[26] C. K. Safeer, E. Jué, A. Lopez, L. Buda-Prejbeanu, S. Auffret, S. Pizzini, O. Boulle, I. M. Miron, and G. Gaudin, Nat. Nanotechnol. 11, 143 (2016).

[27] A. Fert, A. Friederich, and A. Hamzic, J. Magn. Magn. Mater. 24, 231 (1981).

[28] J. J. Prejean, M. J. Joliclerc, and P. Monod, J. Phys. (Paris) 41, 427 (1980). 